\title{
BMJ Open Adolescent mental wellness: a systematic review protocol of instruments measuring general mental health and well-being
}

\author{
Zaida Orth (1) , Brian van Wyk
}

To cite: Orth Z, van Wyk B. Adolescent mental wellness: a systematic review protocol of instruments measuring general mental health and well-being. BMJ Open 2020;10:e037237. doi:10.1136/ bmjopen-2020-037237

- Prepublication history for this paper is available online To view these files, please visit the journal online (http://dx.doi. org/10.1136/bmjopen-2020037237).

Received 24 January 2020 Revised 27 May 2020 Accepted 24 July 2020

\section{Check for updates}

(c) Author(s) (or their employer(s)) 2020. Re-use permitted under CC BY-NC. No commercial re-use. See rights and permissions. Published by BMJ.

School of Public Health, University of the Western Cape, Bellville, South Africa

Correspondence to

Zaida Orth;

zaidaorth@gmail.com

\section{ABSTRACT}

Introduction The promotion of mental health well-being among global adolescent populations is of great public health and social significance. This is particularly true for adolescents living with chronic illnesses as studies have shown that these populations are at higher risk for developing mental health problems. There is vast recognition of the need for age and culturally appropriate interventions to promote mental well-being and prevent mental health problems. In stark contrast, there is a dearth of relevant measures of mental well-being for adolescents. Our proposed systematic review aims to identify measures of mental well-being and to assess content, psychometric properties and relevance to adolescent populations. Methods and analysis The systematic review methodology will be guided by the seven steps proposed by Eggar, Davey and Smith. Documents will be sourced from electronic databases (Academic Search Complete, Educational Resource Information Center, Medical Literature Analysis Retrieval System Online, Cumulative Index of Nursing and Allied Health Literature plus, PsyArticles, SocIndex and Sabinet). All documents will be exported to Mendeley and two reviewers will independently screen the titles, abstracts and full texts for inclusion. Any discrepancies will be resolved by a third party. We will include studies published in all languages from 2000 to 2020, that use an instrument(s) that measure mental well-being among adolescent populations. Studies reporting on clinically significant mental illnesses or disorders will be excluded. A descriptive meta-synthesis approach will be used to identify and describe the mental health instruments used among adolescent populations, and to report on the psychometric properties.

Ethics and dissemination Ethical approval is not required. The results of this review will be disseminated through a peer-reviewed publication as well as conference presentations.

\section{INTRODUCTION}

In recent years, mental health has been identified as a key public health concern, particularly for children and adolescents. ${ }^{12}$ According to $\mathrm{WHO},{ }^{3}$ mental health conditions account for $16 \%$ of the global burden of disease and injury for adolescents aged 10-19 years, with depression being identified as the
Strengths and limitations of this study

- This study contributes to the knowledge around the available and appropriate measures used to measure adolescent mental well-being, which are necessary to develop interventions and inform health policy.

- The search strategy comprises of seven electronic databases to search for peer-reviewed and grey literature.

- A descriptive meta-synthesis approach will be used to describe the mental health well-being components being measured and to assess the psychometric properties of the measurements in the study.

- The limits of this study are related to the time span of the search strategy (2000-2020).

leading cause of disability and illness among this population. Evidence suggests that half of all lifetime mental disorders will start during adolescence, yet the majority of these will go unrecognised and untreated. ${ }^{4}$ There are various factors which hinder the effective treatment of adolescent mental health. These include lack of resources, lack of communication between parents and healthcare practitioners, lack of policies aimed at child and adolescent health, and the ways in which mental health problems are diagnosed. According to Wissow et $a l,{ }^{45}$ many children and adolescents have functional problems related to emotions or behaviour, but do not meet the criteria for diagnosis of a mental health disorder. It is critical though that such at-risk children and adolescents be identified so that appropriate prevention be put in place. This is concerning as adolescence represents a crucial period of development, where exposures, learnt behaviours and experiences can set the trajectory for an individual's mental and physical health in adult life. ${ }^{34}$ The life course approach advocates for effective interventions during adolescence to 
protect public health investments in child survival and early childhood development, and to ensure the physical and mental health and development of the next generation. ${ }^{6}$ To this end, it is imperative to widen the focus from providing care and treatment for adolescents diagnosed with a mental health disorder to include those who are challenged with their mental health and well-being, before diagnoses is reached.

Studies exploring mental health problems among children and adolescents have found that there are various attitudinal, stigma related and structural barriers to accessing mental health services. Findings from a study suggested that $80 \%$ of children and adolescents who are in need of mental health services did not receive any. ${ }^{7}$ These barriers are particularly apparent among adolescents living with a chronic disorder or disease. According to WHO, the incidence and prevalence of chronic conditions is rising in most developed and low/middle-income countries (LMICs) and will constitute the main cause of death for children and adolescents by $2020{ }^{8}$ As such, it is necessary to consider mental health challenges in the context of adolescents living with chronic conditions.

Research has demonstrated that while children and adolescents with chronic health conditions are at increased risk for developing mental health problems, the overall mental well-being of chronically ill youth is largely determined by among others, the severity of the disease, the amount of treatment required and the psychological and social complications associated with such conditions. ${ }^{78}$ The variable nature of these factors have resulted in contradictory findings in different research studies. For example, studies have found gendered differences with chronically ill girls more likely to demonstrate emotional health problems than chronically ill boys when compared with healthy control groups. ${ }^{8}$

Findings from a South African study comparing psychological vulnerabilities among perinatally and behaviourally infected HIV positive adolescents found that perinatally infected adolescents were more likely to be adherent to antiretroviral therapy (ART) and retained in care while behaviourally infected adolescents were more likely to be depressed, report internalised stigma and express suicidal ideation. ${ }^{9}$ These findings suggests that adherence, retention in care and mental health problems among adolescents living with HIV (ALHIV) are differentially influenced by the mode of infection. ${ }^{9}$ As such, tailored interventions are recommended to address the differences between these groups to ensure optimal health outcomes. However, the differences between these two groups may also be influenced by external circumstances related to the care received rather than factors related to the disease. For example, perinatally infected adolescents receive support and build a trusting relationship with their healthcare worker while being in paediatric care. ${ }^{9}$ Behaviourally infected adolescents may not have the similar experiences in the healthcare system.

In the context of prioritising adolescent mental health, prevention and health promotion programmes have been identified as key strategies to help adolescents thrive. ${ }^{3}$ In line with the United Nations Sustainable Developmental Goal 3-which aims to promote well-being for all agesmany countries and organisations are aiming to improve the development of age appropriate interventions to provide psychosocial support and services to adolescents. For example, the Every Child Matters UK government initiative increased investment in child and adolescent mental health services to increase access to appropriate services. ${ }^{10}$ Additionally, global and local policies such as the Global Strategy for Women's, Children's and Adolescents' Health, ${ }^{11}$ the Global Accelerated Action for the Health of Adolescents, ${ }^{12}$ the Lancet Commission on Adolescent Health and Well-being ${ }^{13}$ and the South African National Adolescent and Youth Policy 2017-2022 ${ }^{14}$, have identified adolescent mental health as a priority area and has committed to developing specific programmes to promote mental health among adolescents.

Despite the increased focus on adolescent mental health on the global health agenda, there is a lack of evidence concerning mental health conditions among adolescents, especially in LMICs (AHAH). According to Vreeman, McCoy and Lee, ${ }^{2}$ the lack of information about adolescent mental health in LMICs may be partially related to the lack of validated instruments for these contexts. To address this, UNICEF has launched a project to develop a measure of mental health among adolescents at the population level. ${ }^{3}$ This is of great significance as it will provide valuable evidence regarding the prevalence of mental disorders, inform policy-makers and healthcare workers, and guide intervention and treatment programmes. However, the aim of this instrument is majorly focused on improving data on the prevalence and burden of mental illness, meaning that adolescents who experience challenges to their mental health and well-being, but do not meet the characteristics for a mental diagnosis, may be overlooked.

It is argued that the development of valid instruments to measure mental health are dependent on the ways in which mental health is conceptualised. However, currently, there is little agreement on a general definition of 'mental health' which results in inconsistencies in the way mental health is being measured and addressed in global health policies. ${ }^{15}$ Traditionally, clinical psychologists focused more on the pathogenic side of mental health by describing and treating mental disorders. As such, mental health has popularly been used as a euphemism for 'mental illness'. ${ }^{15}$ Consequently, the majority of the research focused on adolescent mental health adopts a pathological view by focusing on mental health disorders such as psychiatric disorders, general mental health disorders, emotional and behavioural problems, psychological distress and lower levels of illness symptoms as representative of mental well-being. ${ }^{2}{ }^{16}$

However, this pathological view of mental health, and human health in general is not without its critiques. According to WHO, defining human health as more than the absence of illness has been ongoing and elusive 
objective. ${ }^{17}$ As Ryff ${ }^{18}$ argues, to advance our understanding of human health, we should also focus on the presence of wellness and what it means to flourish. In contrast to the pathological view of health, positive psychologists have focused on positive mental health or general mental ${ }^{15}$ health, which is defined not only as the absence of psychopathology, but also the presence of optimal well-being. ${ }^{19}$ From this point, mental health is viewed as including both hedonic (feeling well) and eudemonic (functioning well) traditions of well-being.

The concept of well-being in mental health has gained significant interest as evidence has shown that positive mental health functions as a recovery factor and a protective factor against both physical and mental illness. ${ }^{13} 2021$ Studies have shown that high levels of well-being are associated with better health outcomes in both general and chronic adult populations, while low levels of well-being were associated with poorer health outcomes. ${ }^{20}$ For example, psychological factors, including well-being, has been associated with adjustment in patients diagnosed with hypertension. ${ }^{22}$ Additionally, research has shown that individuals who engage in diverse types of physical activity are more likely to report higher levels of psychological well-being than those who do not exercise, thereby suggesting a link between physical outcomes and well-being. ${ }^{23}$

There are fewer studies focused on adolescent health and psychological well-being. However, the available evidence shows that while adolescents may experience multiple physical, social and emotional changes which can negatively impact their mental well-being, providing psychosocial support and mental health promotion to support positive mental health can protect against psychopathology and support physical health. ${ }^{319}$ For example, a longitudinal study in Australia found that one-third of boys and nearly half of girls in a secondary school experienced an episode of depressive and anxiety symptoms. However, these episodes did not follow into their adult life thereby suggesting that interventions may prevent morbidity in later life. ${ }^{24}$ Additionally, research with ALHIV suggests that mental health well-being may play a role in supporting adherence to ART. A randomised control trial conducted by Willis et $a l^{25}$ found that adolescents who received the community adolescent treatment support intervention where 3.9 times more likely to be adherent to ART in comparison to those who only received standard of care.

While there may be disputes regarding the definition of mental health, there is agreement that mental health should be viewed as more than the absence of mental illness. To this end, WHO has adopted the complete state model to define mental health as 'a state of wellness in which every individual realises his or her own potential, can cope with the normal stresses of life, can work productively and fruitfully, and is able to make a contribution to her or his community, ${ }^{26}$ While this is a step forward in advancing our understanding of mental health in general, considerations should be made that focus specifically on adolescent mental health and its association with physical health, especially given the rise of chronic conditions. Understanding how positive mental health can be used as a resilience resource to protect against psychopathology and poor physical health outcomes is necessary to develop appropriate health policies and interventions. Measures aimed at diagnosing or screening mental health problems are useful in contexts where CAMHS are well developed and supported. However, LMICs like South Africa face challenges relating to lack of policy development and implementation for CAMHs, and a lack of resources and overburdened public healthcare facilities which challenges the sustainability of adolescent mental health and healthcare in general. ${ }^{27}$

Measurements of mental health play a significant role in policy and intervention development as these are used to provide empirical evidence regarding the effectiveness of programmes aimed at improving adolescent mental well-being. Focusing primarily on measuring low level symptoms of a disorder may result in ceiling effects by limiting the range of scores in a positive direction. Consequently, this limits the opportunity to measure the full range of mental health well-being. ${ }^{16}$ Additionally, based on evidence linking well-being and physical health outcomes, it is important to consider these associations in adolescence. Measuring mental health among adolescents necessitates the use of age appropriate and culturally valid instruments that can capture improvements in mental well-being where variances in general mental health can be accurately measured. ${ }^{1628}$

More research focused on emphasising the healthy psychosocial development of adolescents that moves beyond identifying and addressing social and psychological problem trajectories is needed to support the development of psychosocial support programmes and interventions for adolescents who do not qualify for a mental illness diagnosis, yet experience challenges to their mental wellness. ${ }^{29}$ These interventions should be theory driven and accessible within the local culture without placing strain on healthcare systems, especially in resource limited contexts. ${ }^{24} 30$

\section{Rationale for the review}

To identify currently available general mental health and well-being instruments for adolescents between the ages of 10 and 19 years to describe the content and review the psychometric properties of the instruments. For the purpose of this study, general mental health and well-being instruments are those that measure 'generic' outcome measure that does not aim to diagnose and can be applied in a wide range of settings. In other words, these well-being and general mental health factors may include social and psychological functioning, relationships with others, social support, self-perception, quality of life. The findings of this review will provide evidence-based knowledge regarding what mental health instruments are being used in research focused and adolescents, and how reliable and valid these instruments are. 


\section{METHOD AND DATA ANALYSIS}

This section describes the methods that will be used in conducting the systematic review. The seven steps described by Eggar, Davey and Smith ${ }^{31}$ will be used to guide the systematic review process. These steps are: (1) formulate the review question; (2) define the inclusion and exclusion criteria; (3) develop a search strategy; (4) study selection; (5) assess the quality of studies; (6) extract data and (7) analyse or synthesis the data.

\section{Review question}

The following question will guide the review:

1. What instruments are being used to measure/describe mental health outcomes among adolescents?

2. What are the psychometric properties of instruments being used to measure/describe mental health outcomes among adolescents?

\section{Inclusion and exclusion criteria}

Inclusion criteria for the search are as follows:

1. Studies published in peer-reviewed journals or grey literature.

2. The sample includes adolescents between the ages of 10 and 19.

3. The measure used was a self-report measure of general mental health and well-being.

4. Quantitative and mixed-methods studies.

5. Studies published in all languages.

6. Studies from all countries will be included.

7. Studies aimed at developing or validating instruments.

Studies will be excluded based on the following criteria:

1. Review papers or case studies.

2. Screening tools for mental disorders or measures that are disorder/symptom specific.

The decision to include studies with adolescent samples between the ages of 10 and 19 is based on WHO definition of adolescence. However, not all studies focus specifically on adolescents between these age groups. Some studies may include samples of younger children (under 10 years), while other studies may focus on older adolescents and young adults (older than 19 years). Additionally, other studies may focus on mental health for general populations and have adolescents included in the sample. The aim of this study is to review instruments used with adolescents specifically and that recognise adolescence as a unique period of development. Therefore, studies focused on mental health in general populations or young adults (including 18-19years old) will be excluded. Studies that include samples of children younger than 10 may be included if there is a strong focus on adolescent mental health. Furthermore, studies with measures aimed at diagnosing mental health disorders, or that are specific to mental illness are excluded as measures for mental health conditions are well known in comparison to measures of general mental health and well-being. As previously mentioned, many adolescents are often overlooked in mental health policies as they do not meet the requirements for a mental disorder diagnoses. Therefore, we aim to review instruments that can provide a broad measure of general health and well-being that capture a range of common presenting difficulties and strengths.

\section{Search strategy}

The search strategy was developed after consultation with the faculty librarian. The search strategy is broad to include all research articles that use a psychological or psychometric instrument to measure mental health outcomes among adolescents. A systematic database search will be performed using Ebscohost (Psycharticles, Academic Search Premier), Cumulative Index of Nursing and Allied Health Literature (CINAHL), Educational Resource Information Center (ERIC), Medical Literature Analysis Retrieval System Online (MEDLINE) and Sabinet. Full-texts will be searched using the list of key words for the search strategy; "( (adolescen* OR teenage* OR young people OR youth) [AND] (psychological instrument OR measure* OR tool) [AND] (mental health OR mental well-being OR psychological well-being) [AND] (psychometri*; reliability*; validit*)).

\section{Study selection}

Studies were included in the systematic review using the Patient/Problem, Intervention Comparison, Outcome and Time or PICOT (table 1) mnemonics for reviews. These included;

The above-mentioned criteria and search strategy will be used to search the databases. The time period of the search strategy was chosen as due to the paucity of research in this area. ${ }^{2} 1230$ Furthermore, the prioritisation of adolescent health and the focus on adolescent friendly services occurred after the year $2000 .{ }^{32}$ However, we recognise that studies published after 2000 may use measures that have already been developed and that have established psychometric properties. In these cases, we will compare the psychometric data of the study included in the review with the psychometric data available from previous studies if those studies also focused on our population of interest. The screening and reporting of the review will be conducted in accordance with the Preferred Reporting Items for Systematic Reviews and MetaAnalyses guidelines. The number of hits for each database will be recorded and the citations will be exported to Mendeley citation software. Following this, two reviewers will independently review all the titles and abstracts to assess which articles are appropriate for inclusion. The full-text articles of the included abstracts will be downloaded and independently reviewed to determine which articles should be included for the final assessment. ${ }^{31} 33$

\section{Quality assessment}

Evaluating the quality of a study is an essential step in systematic reviews as it ensures validity and reliability of findings. ${ }^{33}$ Therefore, each of the potentially relevant articles to be included in the review will be evaluated using the SFS scoring system (version D) ${ }^{34}$ Version D of the SFS is an appropriate tool to use for assessing the quality studies 
Table 1 Picot based inclusion criteria

\begin{tabular}{ll}
\hline Patient population & Adolescents aged 10-19 years \\
\hline Intervention or Interest & $\begin{array}{l}\text { Reviewing the psychometric properties of psychological tools or instruments which } \\
\text { measure general mental health and well-being among adolescents }\end{array}$ \\
$\begin{array}{ll}\text { Comparison interventions } \\
\text { Outcomes }\end{array}$ & The definitions and/or concepts of mental health and psychological well-being \\
Primary outcomes & Psychometric properties of instrument/measure \\
Secondary outcomes & 2000-2020 \\
Time & Geographical area: global \\
Other considerations & Age limits: adolescents or young adults in the age group 10-19 years. \\
& Language: all language. \\
& Study designs: quantitative method or mixed methods. \\
\hline
\end{tabular}

in this review as it will allow the two reviewers to assess the appropriateness of the methodological elements of the included studies, such as the psychometric properties of the instruments and the theoretical and operational definitions used to define constructs. The SFS version D scoring systems contains 29 questions covering the following subsections: (1) purpose of the measure which includes questions regarding the purpose, target group and theoretical dimensions of the measure; (2) methodological rigour which focuses on the design, sample, data collection and analyses; and (3) general considerations which includes questions regarding the type of publication. The first reviewer will score each of the included studies using the scoring system and presented in tabular form to be reviewed by the second reviewer. The overall quality of the study is based on the score it obtained and will be categorised as either weak $(0 \%-25 \%)$, moderate $(26 \%-50 \%)$, strong $(51 \%-75 \%)$ or excellent $(76 \%-$ $100 \%$ ). Only articles with a score of $51 \%$ and above will be included in the analysis.

\section{Data extraction}

Following the quality assessment, a data extraction sheet will be set up in excel. The two reviewers will then extract the sample characteristics (ages, gender, school grade, etc) geographical location, mental health concepts, psychometric properties of the instrument, type of instrument and the format of the instrument and the results of the instrument. For the purpose of this study, only data presented in the articles will be used as we are interested in how the data is reported. Where needed, we will contact authors for original data.

\section{Data synthesis}

A descriptive meta-synthesis approach will be used to identify and describe the mental health instruments used among adolescent populations, and to report on the psychometric properties. The synthesis of information regarding each instrument will be presented in tabular form in which the included articles will be ranked based on the methodological rigour scores of the quality assessment. ${ }^{35}$ This will allow the reviewers to assess whether the research design used reflects the aims and objectives of a particular study and whether the conclusions are supported by the data. ${ }^{33}$ As part of the aims of the review, we will focus on how mental health or concepts of mental health are being defined in the studies and which theories are being used (if any) to frame mental health. As we are looking at sample characteristics, we will consider if there are any notable differences in the studies based on sample characteristics. We would also be interested to see what the differences are across countries regarding the number of studies being conducted in higher and LMICs, and how mental health is investigated in these contexts. The data extracted from each of the included articles will be presented in the table to clearly present a summary of the core findings.

\section{Patient and public involvement}

As this is a protocol for a systematic review, no patients or public were involved in the design or research of this study.

\section{ETHICS AND DISSEMINATION}

Ethics approval is not required as the systematic review does not involve the participation of human subjects; rather it involves reviewing and collecting data from publicly available sources.

This review aims to contribute to research on adolescent mental health. Specifically, it aims to understand what measures are available to measure mental health and well-being to identify gaps and areas of improvement for future measures. There is an increasing need to develop instruments, which can measure all aspects of adolescent mental health. These include instruments which can identify adolescents who may be experiencing challenges to their mental health, yet do not qualify for a mental illness diagnosis. Measures of general mental health and well-being may provide useful information to inform intervention and policy. We plan to publish the 
results of this systematic review and present the findings to key stakeholders and colleagues on various platforms such as webinars and conferences.

Contributors Z0 conceived the idea, developed the research question and study methods and contributed meaningfully to the drafting and editing; she also approved the final manuscript. BvW aided in developing the research question and study methods, contributed meaningfully to the drafting and editing, and approved the final manuscript.

Funding ZO is supported by the National Research Foundation, grant number (118160). Additionally, the authors would like to acknowledge funding form the Belgian Development Cooperation, through the Institute of Tropical Medicine Antwerp. Any opinion, finding and conclusion or recommendation expressed in this material is that of the authors and not the funders.

Disclaimer Any opinion, finding and conclusion or recommendation expressed in this material is that of the authors and not the funders.

Competing interests None declared.

Patient and public involvement Patients and/or the public were not involved in the design, or conduct, or reporting, or dissemination plans of this research.

Patient consent for publication Not required.

Provenance and peer review Not commissioned; externally peer reviewed.

Open access This is an open access article distributed in accordance with the Creative Commons Attribution Non Commercial (CC BY-NC 4.0) license, which permits others to distribute, remix, adapt, build upon this work non-commercially, and license their derivative works on different terms, provided the original work is properly cited, appropriate credit is given, any changes made indicated, and the use is non-commercial. See: http://creativecommons.org/licenses/by-nc/4.0/.

ORCID iD

Zaida Orth http://orcid.org/0000-0002-2895-0417

\section{REFERENCES}

1 World Health Organization. Health for the world's adolescents: a second chance in the second decade.

2 Vreeman RC, Mccoy BM, Lee S. Review article mental health challenges among adolescents living with HIV 2017;20:100-9.

3 World Health Organization. Adolescent mental health, 2018. Available: https://www.who.int/news-room/fact-sheets/detail/ adolescent-mental-health

4 Wissow L, Anthony B, Brown J, et al. A common factors approach to improving the mental health capacity of pediatric primary care. Adm Policy Ment Health 2008;35:1-7.

5 UNICEF Measurement of Mental Health among Adolescents at the Population Level (MMAP). Conceptual framework and roadmap the roadmap to the measurement of mental health.

6 World Health Organization. Mental health action plan 2013-2020. World health organisation. Geneva: Production Services, 2013.

7 Delamater A, Delamater AM, Guzman A, et al. Mental health issues in children and adolescents with chronic illness 2017.

8 Michaud P-A, Suris J-C, Viner R. The adolescent with a chronic condition: epidemiology, development issues and health care provision 2007.

9 Sherr L, Cluver LD, Toska E, et al. Differing psychological vulnerabilities among behaviourally and perinatally HIV infected adolescents in South Africa - implications for targeted health service provision. AIDS Care 2018;30:92-101.

10 Thornton H. Every child matters. J Diabetes Nurs 2007;11:222-3.
11 United Nations. The Global Strategy for Women's, Children's and Adolescents' Health (2016-2030) 2016:41.

12 World Health Organization. Global accelerated action for the health of adolescents (AA-HA!) guidance to support country implementation. World Health Organization, 2017.

13 Patton GC, Sawyer SM, Santelli JS, et al. Our future: a Lancet Commission on adolescent health and wellbeing. Lancet 2016;387:2423-78

14 National Department of Health. National adolescent \& youth health policy 2017:31.

15 Manwell LA, Barbic SP, Roberts K, et al. What is mental health? Evidence towards a new definition from a mixed methods multidisciplinary international survey. BMJ Open 2015;5:1-11.

16 Rose T, Joe S, Williams A, et al. Measuring Mental Wellbeing Among Adolescents : A Systematic Review of Instruments. 2017:2349-62.

17 Svalastog AL, Donev D, Jahren Kristoffersen N, et al. Concepts and definitions of health and health-related values in the knowledge landscapes of the digital Society. Croat Med J 2017;58:431-5.

18 Ryff CD. Psychological well-being revisited: advances in the science and practice of eudaimonia. Psychother Psychosom 2014;83:10-28.

19 Keyes CLM. Promoting and protecting mental health as Flourishing 2005:95-108

20 Trompetter HR, Ten Klooster PM, Schreurs KMG, et al. Measuring values and committed action with the engaged living scale (ELS): psychometric evaluation in a nonclinical sample and a chronic pain sample. Psychol Assess 2013;25:1235-46.

21 Ryff CD, Singer BH, Dienberg Love G. Positive health: connecting well-being with biology. Philos Trans $R$ Soc Lond B Biol Sci 2004;359:1383-94.

22 Rafanelli C, Offidani E, Gostoli S, et al. Psychological correlates in patients with different levels of hypertension. Psychiatry Res 2012;198:154-60.

23 Edwards SD, Ngcobo HS, Edwards DJ, et al. Exploring the relationship between physical activity, psychological well-being and physical self- perception in different exercise groups. South African $J$ Res sport Phys Educ Recreat 2005;27.

24 Sawyer MG, Arney FM, Baghurst PA, et al. The mental health of young people in Australia : key findings from the child and adolescent component of the national survey of mental health and well-being 2001.

25 Willis N, Milanzi A, Mawodzeke M, et al. Effectiveness of community adolescent treatment supporters (cats) interventions in improving linkage and retention in care, adherence to art and psychosocial well-being: a randomised trial among adolescents living with HIV in rural Zimbabwe. BMC Public Health 2019;19:1-9.

26 WHO. Mental health: a state of well-being 2019.

27 Mokitimi S, Schneider M, de Vries PJ. Child and adolescent mental health policy in South Africa: history, current policy development and implementation, and policy analysis. Int J Ment Health Syst 2018;12:1-15.

28 Bentley N, Hartley S, Bucci S. Systematic review of self-report measures of general mental health and wellbeing in adolescent mental health. Clin Child Fam Psychol Rev 2019;22:225-52.

29 Rose T, Shdaimah C, De TD, et al. Children and youth services review exploring wellbeing and agency among urban youth through photovoice. Child Youth Serv Rev 2016;67:114-22.

30 Sherr L. Mental health - a bridge not so far. The Lancet Glob Heal 2017;5:e559-60.

31 Eggar M, Smith GD, Altman DG. Systematic reviews in health care: meta analysis in context. 2nd ed. London: BMJ Publishing Group, 2001.

32 World Health Organization. Adolescent friendly health services 2011;46:1-3.

33 Gough D, Oliver S, Thomas J. Systematic reviews.

34 Smith M, Franciscus G, Swartbooi C, et al. The SFS scoring system.

35 Thomas J, Harden A. Methods for the thematic synthesis of qualitative research in systematic reviews. BMC Med Res Methodol 2008;8:1-10. 